ROCZNIKI HUMANISTYCZNE

Tom LXVIII, zeszyt 5 - 2020

DOI: http://dx.doi.org/10.18290/rh20685-5

\title{
BENJAMIN STEINS DIE LEINWAND: RÜCKKEHR JÜDISCHER RELIGIOSITÄT IM ZEICHEN EINER PREKÄR GEWORDENEN ERINNERUNG AN DIE SHOAH
}

\begin{abstract}
A b strakt. Benjamin Steins Roman Die Leinwand fiktionalisiert den Fälschungsskandal um Binjamin Wilkomirski, dessen als Zeugnis der Shoah ausgewiesener Text sich als fiktive Erzählung entpuppte. Die Leinwand problematisiert dabei provokativ die Zuverlässigkeit von Erinnerungen und hinterfragt die Grenze zwischen Autobiographie und Fiktion. Der Aufsatz argumentiert, dass diese ästhetischen Strategien vor dem Hintergrund des Sterbens der letzten ZeugInnen sowie einer dadurch notwendigerweise mediatisierten Erinnerung an die Shoah interpretiert werden müssen. Im Zeichen dieser Entwicklungen findet im Roman eine Hinwendung zum religiösen Aspekt jüdischer Identität statt. Ihr kritisches Potential mit Blick auf den Erinnerungsdiskurs entwickelt Die Leinwand dabei vor allem durch die literarische Reflexion postmoderner Theoriebildungen.
\end{abstract}

Schlüsselwörter: Benjamin Stein; Erinnerungskultur; kollektives Gedächtnis der Shoah; jüdische Religiosität; Postmoderne.

\section{DIE LEINWAND, WILKOMIRSKI UND DIE ROLLE DER ERINNERUNG AN DIE SHOAH}

Lange Zeit galt jüdisches Schreiben nach der Shoah in Deutschland von der viel zitierten „negativen Symbiose“ geprägt, jener ,gegensätzliche[n] Gemeinsamkeit“, die Dan Diner zufolge das Verhältnis zwischen Deutschen und Juden seit Auschwitz unweigerlich bestimme (Diner 185). Nach Andreas B. Kilcher ergaben sich für die Literatur der NachfahrInnen der Überlebenden hieraus zwei verschiedene Formen der Exterritorialität: Die eine sei durch die tatsächliche, geographische Emigration deutsch-jüdischer Auto-

FABIAN SADER, M.A. - Europa-Universität Viadrina, Kulturwissenschaftliche Fakultät; Korrespondenzadresse: Große Scharrnstraße 59, D-15230 Frankfurt (Oder); E-Mail: euv06374@europauni.de. ORCID: https://orcid.org/0000-0002-2591-2933. 
rInnen charakterisiert, die andere wiederum durch das Spezifikum eines ästhetischen Avantgardismus, der sich von den traditionellen Normen der Literatur bewusst abhebe und solchermaßen jener „negativen Symbiose“ aussetze (Kilcher 133-34). Mit der Wendezeit aber hat sich der Rahmen für heutiges jüdisches Schreiben in Deutschland grundlegend geändert: Der Zusammenbruch des Ostblocks ermöglichte die Einwanderung von ca. 200.000 sogenannten „Kontingentflüchtlingen“, die ihre eigenen und jeweils sehr verschiedenen Erinnerungsnarrative mitbrachten. Diese entsprachen nicht mehr jenen der bereits zuvor in Deutschland lebenden und schreibenden Jüdinnen und Juden. Mit Blick darauf spricht Jakob Hessing von einem „Doppelantlitz des deutsch-jüdischen Verhältnisses“, das sich auch für die deutsch-jüdische Literatur nachweisen lasse: Während für den einen Teil die Erinnerung an die Shoah noch im Mittepunkt stehe, spiele diese für den anderen hingegen keine entscheidende Rolle mehr (Hessing 244-45). Daneben begannen nun auch Jüdinnen und Juden aus der ehemaligen DDR damit, sich ihrem Judentum wieder zuzuwenden (253). Im Kontext solcher Entwicklungen schlussfolgern Katja Garloff und Agnes Mueller, dass die These von einer „negativen Symbiose" dem komplexer gewordenen Binnenverhältnis von deutschen Jüdinnen und Juden und nicht-jüdischen Deutschen heute kaum noch gerecht werde (Garloff und Mueller 5). Auch Andree Michaelis-König fasst zusammen, dass, inmitten seiner Vielfältigkeit, stets zwei grundsätzliche Charakteristiken deutschsprachig-jüdischen Schreibens erkennbar gewesen seien, nämlich der Orientierungspunkt jüdischer Kultur vor der Shoah einerseits sowie das (Selbst-)Verständnis, Überlebende oder deren NachfahrInnen zu sein andererseits - mit Blick auf die jüngeren AutorInnen treffe dies in Teilen zumindest nicht mehr zu (Michaelis-König 146-47).

Vor diesem Hintergrund erweist sich der Versuch einer Einordnung von Benjamin Steins Die Leinwand zunächst als schwierig. Das Thema der Erinnerung an die Shoah ist in ihm durchaus präsent, allerdings auf ungewöhnliche Weise: Der Roman des in Ostdeutschland aufgewachsenen Autors rückt den Fälschungsskandal um Binjamin Wilkomirski in den Mittelpunkt. Dessen 1995 erschienenes Buch Bruchstücke. Aus einer Kindheit 1939-1948 war als die persönlichen Erinnerungen des Autors angekündigt worden, die zu einem Großteil von seinen schrecklichen Erfahrungen während der Shoah erzählen sollten. Es stellte sich jedoch drei Jahre später heraus, dass der Text kein Zeugnis, sondern lediglich eine fiktive Erzählung war. Das Buch verschwand daraufhin wieder vom Markt. Es entspann sich im Nachgang eine Debatte, die aufs Neue die grundsätzliche Möglichkeit von Authentizität der 
ZeugInnenberichte problematisierte (Kilian 282-84). Die Leinwand wirft nun mehr als ein Jahrzehnt nach dem Skandal um Bruchstücke tatsächlich die Frage auf, ob der Geschichte Wilkomirskis bzw. Minskys - so der Name des fiktionalisierten Pendants im Roman - nicht ebenso eine Form von Berechtigung, eine eigene Form von Wahrheit zugestanden werden könne. Mittels dieser Geste aber scheint Steins Roman im Umkehrschluss an der Autorität der ZeugInnen der Shoah zu rütteln. Mit Blick darauf könnte er somit ,als paradigmatisch für eine literarische Verabschiedung des Zeugenparadigmas gelesen werden“ (Horstkotte 117). Eine weitere Besonderheit von Die Leinwand besteht zudem darin, dass der Text zu einem großen Teil den Lebensalltag zweier jüdisch-orthodoxer Protagonisten darstellt. Denn eine deutschjüdische Literatur, die als tatsächlich religiös einzuordnen ist, existiert in der Moderne und im Grunde über das 20. Jahrhundert hinweg nicht (Hoffmann 435). Teile der Forschung gelangen deshalb zu dem Schluss, dass mit Benjamin Stein der religiöse Aspekt jüdischer Identität zu Beginn des 21. Jahrhunderts in die Literatur zurückkehrt (Kleine 86; Garloff 141), während die Erinnerung an die Shoah bei ihm dagegen in den Hintergrund rücke (Kleine 133; Koelle 227; Schuchmann 219-20).

Es wird hieraus allerdings keinesfalls ersichtlich, weshalb dann ausgerechnet der Skandal um ein gefälschtes Zeugnis der Shoah, der immerhin eine breite Diskussion über die Rolle der Erinnerungskultur in Deutschland nach sich zog (Oels 377), als der eigentliche Aufhänger für Die Leinwand dient. Im Folgenden soll deshalb gezeigt werden, dass Steins Roman weniger eine Rückkehr jüdischer Religiosität auf Kosten der Erinnerung an die Shoah darstellt, sondern demgegenüber vielmehr als eine Hinwendung zum Religiösen im Zeichen einer prekär gewordenen Erinnerung gelesen werden kann. Auf diese Weise kann Die Leinwand vor allem im Kontext eines sich fundamental wandelnden kollektiven Gedächtnisses betrachtet werden: „Vier Argumente - Generationswechsel, Gedächtnis-/Medienwechsel, Sprachwechsel und gesellschaftlicher Wandel - stellen die Grundlage für die Transformation des Gedächtnisparadigmas“ (Frieden 59). In Hinblick darauf sollen im weiteren Verlauf zunächst einige der zentralen ästhetischen Strategien in Steins Roman in den Fokus rücken, welche die Unzuverlässigkeit von Erinnerungen vorführen und die Grenzen zwischen Autobiographie und Fiktion problematisieren. Jene Strategien sollen im Anschluss daran an ein sich veränderndes kollektives Gedächtnis der Shoah rückgekoppelt werden. Auf diese Weise soll die Funktion des Religiösen im Roman ersichtlich werden, das in einem Moment der daraus möglicherweise resultierenden Verunsicherung 
für das jüdische Selbstverständnis ein alternatives Identitätsangebot ermöglicht. Abschließend soll in aller Kürze die komplexe, ästhetische Reflexion postmoderner Theoriebildungen angedeutet werden, auf welcher Die Leinwand dabei gründet und die erst das eigentliche Fundament für ihre kritische Befragung des Erinnerungsdiskurses um die Shoah stellt.

\section{ERINNERUNG UND AUTOBIOGRAPHIE - ALLES NUR FIKTION?}

Bereits die grundsätzliche Aufmachung von Die Leinwand sticht hervor: Der Roman ist als ein von zwei Seiten lesbarer Text angelegt, dessen zwei Teilerzählungen Amnon Zichroni und Jan Wechsler sich in der Mitte des Buches treffen. Da der Paratext explizit keine Lektüreempfehlungen vorgibt, müssen sich die LeserInnen jeweils individuell für einen Romananfang entscheiden. Auch im Fortgang der Lektüre bleibt es ihnen überlassen, ob sie die beiden Erzählungen nacheinander lesen oder nach den einzelnen Kapiteln zwischen ihnen jeweils hin- und herspringen. Jede Entscheidung aber verändert zu einem gewissen Grad letztlich auch die konkrete Leseerfahrung.

Amnon Zichroni, dessen Nachname im Hebräischen für „mein Gedächtnis“ (Horstkotte 119) steht, erzählt rückblickend davon, wie er in einem streng-orthodoxen jüdischen Umfeld in Jerusalem aufwächst. Da er in der Jeschiwa beim Lesen weltlicher Literatur erwischt wird, schickt ihn sein Vater zu einem alten Freund in die Schweiz. Dieser weist Zichroni in die Vergnüglichkeiten der Literatur ein, lehrt ihn aber gleichermaßen die Notwendigkeit religiöser Observanz und Skepsis gegenüber den „rationalen“ Wissenschaften. Zichroni geht schließlich in die USA, um dort den Weg der religiösen Erziehung weiter zu verfolgen. Er entwickelt die übernatürliche Gabe, mittels eines Blicks oder einer Berührung in die Erinnerungen anderer Menschen eintauchen und diese physisch gar selbst durchleben zu können. Hieraus erwächst sein Wunsch, sich zum Psychologen ausbilden zu lassen. Durch das intensive Studium ethisch-religiöser Texte versucht er, den Einsatz seiner Gabe besser kontrollieren zu können. Nach dem Tod seines Ziehvaters trifft Zichroni in der Schweiz schließlich auf den Geigenbauer Minsky, von dem er eine geerbte Violine reparieren lassen möchte. Es stellt sich allerdings heraus, dass Minsky von schrecklichen Erinnerungen aus dem Vernichtungslager Majdanek geplagt wird. Zichroni verzichtet auf ein komplettes Eintauchen in Minskys Gedächtnis, steht ihm allerdings zur Seite, als 
er seine Erinnerungen in Form eines Buchs unter dem Titel Aschentage publiziert und dadurch Berühmtheit erlangt. Doch die scheinbare Autobiographie Minskys entpuppt sich bald darauf als fiktive Erzählung. Zichroni muss seinen Beruf als Psychologe aufgeben. Er zieht sich schlussendlich in eine jüdische Siedlung im Westjordanland zurück.

Der Protagonist der anderen Teilerzählung, Jan Wechsler, ist wiederum für die Aufdeckung von Minskys mutmaßlich gefälschtem Zeugnis verantwortlich. Kurioserweise ist er sich dessen zu Beginn jedoch keinesfalls bewusst. Der ebenso orthodoxe Jude Wechsler erzählt im Gegensatz zu Zichroni aus der Gegenwart heraus, gleichwohl mit zahlreichen Analepsen versetzt. An Schabbes erhält er einen mysteriösen Koffer mit ihm unbekannten Gegenständen, welche die LeserInnen des anderen Teils bereits als Zichroni zugehörig erkennen. Vor allem ein Buch mit dem Titel Maskeraden, das sich mit der Aufdeckung des Minsky-Falls befasst, bereitet ihm Kopfzerbrechen: Er kann sich nicht erklären, weshalb dessen Autor gerade seinen Namen trägt. Im Fortgang der Erzählung berichtet Wechsler von seiner Jugend in der ehemaligen DDR und seinem schwierigen Weg zum Judentum. Dabei stellt sich jedoch zunehmend heraus, dass diese Vergangenheit eine erfundene sein muss und er offensichtlich falschen Erinnerungen aufsitzt. Sein Leben gerät aus den Fugen, die Familie verlässt ihn. Gleichzeitig ahnt Wechsler, dass ausgerechnet er derjenige gewesen sein muss, der Minsky öffentlich aufgrund seiner unwahren Erinnerungen anprangerte. In der Hoffnung, sein verlorengegangenes Ich wiederzufinden, begibt er sich schließlich nach Israel: Er glaubt, im Laufe seines letzten Besuchs dort möglicherweise sein Gedächtnis verloren zu haben. Nach seiner Ankunft am Flughafen Ben Gurion wird Wechsler allerdings sofort verhaftet, weil man ihn im Zusammenhang mit dem Verschwinden von Amnon Zichroni verdächtigt. Er erinnert sich schließlich wieder an seine letzte Reise nach Israel, während der es tatsächlich zu einem Kampf mit Zichroni an einer Mikwe gekommen war. Hier, an dem rituellen Tauchbad, prallen die beiden Erzählungen also gewissermaßen aufeinander.

Gleich zu Beginn der Amnon Zichroni-Teilerzählung kündigt sich das den Roman inhaltlich sowie formal durchziehende Thema der Unzuverlässigkeit von Erinnerungen an:

Erinnerung aber ist unbeständig, stets bereit, sich zu wandeln. Mit jedem Erinnern formen wir um, filtern, trennen und verbinden, fügen hinzu, sparen aus und ersetzen so im Laufe der Zeit das Ursprüngliche nach und nach durch die Erinnerung an die Erinnerung. Wer wollte da noch sagen, was einmal wirklich geschehen ist? (Stein Z.7-8) 
Diese Worte sind selbstverständlich als ein Vorgriff auf den im Roman fiktionalisierten Wilkomirski-Skandal zu lesen. Dem individuellen Gedächtnis grundsätzlich eine faktengetreue Wiedergabe der Vergangenheit zuzutrauen, erschiene naiv. Stattdessen reflektiert Die Leinwand hier bereits Erkenntnisse aus Teilen der Autobiographie-Forschung, die den Erinnerungsprozess notwendigerweise ,als eine kreative Leistung, als nachträglicher konstruktiver Akt und damit zwangsläufig als Bruch mit der Vergangenheit“ (Düwell 19) betrachten. Schon die titelgebende Metapher der Leinwand verweist dabei auf den prinzipiell selektiven und somit konstruktiven Charakter individueller Erinnerungen, was sich ganz besonders in der Beschreibung der psychoanalytischen Methode der Übertragung durch Zichroni zeigt:

In der Analyse konnte man ihnen [den PatientInnen, Anm. F.S.] die Zügel wieder in die Hand geben - oder vielmehr die Palette und den Pinsel, mit dem sie auf der Leinwand ihrer Erinnerungen neue Akzente setzten. Dabei konnte man selbst ganz zur Leinwand werden, zu einer Projektionsfläche, auf der die Patienten mögliche Gegenentwürfe skizzierten und neue Möglichkeiten erprobten [...]. (Stein Z.152)

Erinnerungen sind nach dem Erzähler - um im semantischen Feld der Malerei zu verbleiben - über- und ebenso unterschiedlich malbar, sodass es also fraglich bleibt, ob die oberste Schicht des Gezeichneten tatsächlich noch das eigentlich zu Erinnernde darstellen kann. Gerade für die Gesundung traumatisierter PatientInnen erscheint die Fähigkeit dieser Gestaltbarkeit des individuellen Gedächtnisses nämlich elementar. Dies gilt umso mehr, da Zichroni Erinnerung als identitätskonstituierend begreift: „Unsere Erinnerungen sind es, die uns zu dem machen, was wir sind. Unser Gedächtnis ist der wahre Sitz unseres Ichs“ (Z.7). Im Aufzeigen dieses Nexus folgt Die Leinwand einem durchaus häufig behandelten Sujet in der Gegenwartsliteratur (Neumann 149). Minskys alias Wilkomirskis vermeintliches Erinnern an Majdanek half laut Zichroni dabei, dass ersterer „Stück für Stück seine Identität wiederfand" (Stein Z.176). Der tatsächliche Wahrheitsgehalt des Erinnerten scheint dabei zunächst erst einmal keine Rolle zu spielen, da die Trennlinie zwischen Erinnerung und Fiktion ohnehin unscharf ist. Für Minsky persönlich aber sei dessen Erzählung aufgrund ihrer identitätsstiftenden Funktion überlebenswichtig gewesen (Z.176).

Reflektiert die Erzählung Amnon Zichroni vor allem auf inhaltlicher Ebene das fiktionale Moment innerhalb einer jeden Erinnerung, erweist sich ebendies als kennzeichnendes narratives Merkmal der anderen Geschichte 
Jan Wechsler. Diese sticht besonders durch einen unzuverlässigen Erzähler hervor, der im Erzählvorgang als vergesslich erscheint, sich widerspricht und retrospektiv von einer erdachten und nie stattgefundenen Vergangenheit berichtet. Das stete Changieren zwischen der gegenwärtigen Erzählebene und den zahlreichen Analepsen kann dabei als eine literarische „Mimesis des Erinnerns" (Basseler und Birke 127) bezeichnet werden, welche die Unzuverlässigkeit von Erinnerungen im Prozess der Narration gewissermaßen vorführt: So finden sich zahllose Textkommentare wie „Daran konnte ich mich nicht erinnern.“ (Stein W.10), „Sicher bin ich mir nicht.“ (W.17) oder „Eigentümlich. Ich kann mich für gewöhnlich auf mein Gedächtnis verlassen“ (W.18), wenn Wechsler bestimmte Ereignisse im Rückblick entfallen. Jene Unzuverlässigkeit wird hier freilich drastisch zugespitzt, da Wechslers (Selbst-)Erzählung nicht nur eine an manchen Stellen abweichende, durch Sprache vermittelte Wiedergabe des Geschehenen darstellt, sondern demgegenüber keinerlei Bezug zu einem tatsächlichen Referenten aufweist. Dies aber rückt den Kläger Wechsler unweigerlich in die Nähe zum Angeklagten Minsky alias Wilkomirski, weshalb Wechslers rhetorische Frage als durchaus stellvertretend für diesen formuliert wirkt: „Aber macht mich der Umstand, dass ich mich gelegentlich irre und sich meine Erzählungen nicht mit den beweisbaren Fakten in Einklang bringen lassen, zu einem Lügner?“ (W.27) Mittels dieser ironischen Geste problematisiert Die Leinwand den grundsätzlichen Anspruch auf Authentizität, der an Autobiographien gemeinhin gestellt wird und der so auch konkret an Wilkomirskis Bruchstücke als das vermeintliche Zeugnis eines Überlebenden der Shoah gestellt wurde. Es ist hier die Rede vom sogenannten autobiographischen Pakt, den Philippe Lejeune zufolge die LeserInnen mit einem Text eingehen, sobald sie von einer Identität zwischen AutorIn, ErzählerIn und ProtagonistIn ausgehen können (Lejeune 27-28). Die Beschreibungen des Paratexts von Bruchstücke ließen seinerzeit gar keinen anderen Schluss zu, als dass jene Identität in diesem Falle unweigerlich gegeben sein müsste. Wie illusorisch jener autobiographische Pakt aber sein kann, führt Die Leinwand anhand einer Art paratextuellen Spiels vor: Die sich im Laufe der Erzählung zunehmend als phantasiert herausstellende Vergangenheit der literarischen Figur Jan Wechslers entpuppt sich nämlich mehr und mehr als Teil der tatsächlichen Biographie des realen Autors Benjamin Stein, wie bereits ein flüchtiger Blick auf den Paratext von Die Leinwand zeigt. Darüber hinaus erweist sich jener Debütroman, auf den Wechsler im Laufe seiner Recherchen zu seinem vermeintlichen Namensvetter bzw. Autor des Buchs Maskeraden stößt, als auf- 
fallend ähnlich zu Steins realem Erstlingswerk Das Alphabet des Juda Liva und beinhaltet obendrein auch noch Elemente aus der Familiengeschichte von Benjamin Stein (W.78-80). Die Schlussfolgerung des verwirrten Wechsler über seinen mutmaßlichen Doppelgänger gerät schließlich zu metafiktionaler Ironie: „Was immer er in seinem Roman erzählt, kann nur Fiktion sein und keinesfalls autobiographisch" (W.80). Denn so wie autobiographische Erzählungen notwendigerweise fiktionale Elemente beinhalten, kann die Fiktion umgekehrt gleichermaßen in Teilen auf autobiographischen Momenten gründen: Hierin erscheint Die Leinwand stellenweise als literarische Reflexion der Einsicht Paul de Mans, wonach die Autobiographie eben keinesfalls eine Textgattung sei, ,sondern eine Lese- und Verstehensfigur, die in gewissem Maße in allen Texten auftritt" (de Man 134). Daraus aber, dass nach de Man nur die „Illusion der Referenz“ existiert bzw. der Bezug auf diese „nur eine Figuration unter anderen“ darstellt, ergibt sich letztlich: „Die Unterscheidung zwischen Fiktion und Autobiographie scheint also keine Frage von Entweder-Oder zu sein, sondern unentscheidbar" (133). Ebendies scheint Die Leinwand vermittels des versatzstückartigen Einbaus der „echten“ Biographie Benjamin Steins, die sich ausgerechnet für die literarische Figur Jan Wechsler als fiktiv erweist, mit Blick auf den Skandal um Wilkomirski vorführen zu wollen. Die Frage nach der Authentizität von dessen Erinnerungen erscheint solchermaßen schlechterdings irrelevant.

Die auffällige Form von Steins Roman scheint dabei in engem Zusammenhang mit jener Auffassung der „Illusion der Referenz“ zu stehen: So erweist sich der Lesevorgang geradezu als Inszenierung der unhintergehbaren Fiktionalität innerhalb einer jeden nachträglichen Versprachlichung. Der Inhalt von Die Leinwand lässt sich nämlich stets auf andere Weise nacherzählen, nicht nur aufgrund der für den literarischen Text generell charakteristischen Unbestimmtheiten, sondern darüber hinaus durch die jeweiligen Entscheidungen der LeserInnen im Verlaufe des Lektüreprozesses. So werden beispielsweise bestimmte Motive erst dann sichtbar, wenn man sich nach den einzelnen Kapiteln jeweils für das Hinüberwechseln in die andere Teilerzählung entscheidet. Im sechsten Kapitel von Amnon Zichroni und Jan Wechsler ist es z.B. das Motiv des Zufalls, das den weiteren Verlauf der zwei Erzählungen prägt und die beiden Protagonisten schließlich zu ihrer jeweiligen Begegnung mit Minsky führt (Stein W.90-92, Z.88-91). Würde man beide Teilerzählungen hingegen jeweils in einem Stück lesen, bliebe jenes Motiv mit hoher Wahrscheinlichkeit größtenteils unbemerkt. Ebenso ist es keinesfalls unwichtig, mit welcher der beiden Erzählungen die LeserInnen 
ihre Lektüre beginnen: Der Übergang von Amnon Zichroni zu Jan Wechsler funktioniert gewissermaßen fließend, die Mikwe scheint hier als Motiv des Identitätswechsels zu fungieren und Zichroni, aufgrund seiner übernatürlichen Gabe, während des Kampfs zwischen den Protagonisten sich im Gedächtnis Wechslers zu verlieren (Z.193). Es ließe sich somit annehmen, dass dies der eigentliche Grund für Wechslers Gedächtnisverlust sein könnte. Anders herum - von Jan Wechsler zu Amnon Zichroni - bildet die Mikwe dagegen eine Sackgasse, und Wechsler springt letztlich in ein leeres Tauchbecken (W.204). Wollte man die beiden Erzählungen in dieser Reihenfolge nacherzählen, ergäbe sich in der Mitte zwischen ihnen also ein größerer Bruch. Auf diese Weise simulieren die LeserInnen hier anhand ein und desselben literarischen Texts durch ihre Selektionen die Konstruktion von Bedeutung, die nicht zuletzt auch die autobiographische Erzählung vornehmen muss. Dadurch aber kann sie dem Anspruch nach einer mimetischen Abbildung des Geschehenen bzw. zu Erinnernden gar nicht gerecht werden.

Lässt sich die Autobiographie auch nicht auf einen ausschließlich dokumentarischen Charakter reduzieren, mag man dennoch kaum bestreiten, dass ihr nichtsdestotrotz ein auf unbestimmte Weise vermittelter Bezug zu einem außersprachlichen Referenten und folglich ein wenigstens ,hybrider Charakter als Textsorte zwischen Fakt und Fiktion" (Finck 12) innewohnen muss. Sie ist zwischen der Geschichte und der Literatur, ,an den Grenzen der Diskurse" (Michaelis 52) angesiedelt und als solche nicht genau verortbar. Gerade in den autobiographischen Erzählungen Überlebender der Shoah „reflektieren die Autorinnen und Autoren [...] den Grad der Fiktionalisierung sowie die Fallen des Erzählens“ (Günter 12-13). In Die Leinwand können Jan Wechsler und Minsky alias Wilkomirski diese Ambivalenz jedoch keinesfalls für sich beanspruchen, da ihre Geschichten sich als komplett fiktiv erweisen. Es stellt sich also die Frage, wohin die Problematisierung der Grenzen von Autobiographie und Fiktion anhand der Fiktionalisierung des Skandals um Wilkomirski hier führt: Spielt es in Steins Roman tatsächlich keine Rolle mehr, ob einer autobiographischen Erzählung über die Shoah ein tatsächlicher Wahrheitsgehalt zugrunde liegt oder nicht? Solchermaßen wäre die epistemische sowie auch die soziale Kompetenz, auf der sich die Autorität von ZeugInnen im Allgemeinen gründet (Schmidt und Voges 11-12), schlechterdings infrage gestellt. Gehört Die Leinwand damit jenem Teil der jüngeren deutschsprachig-jüdischen Literatur an, für den das Selbstverständnis, zu den NachfahrInnen der Überlebenden der Shoah zu gehören, nicht länger im Mittelpunkt steht? 


\section{DIE FRAGE NACH EINER VERANTWORTUNGSVOLLEN WEITERGABE DER ERINNERUNG AN DIE SHOAH UND DIE SUCHE NACH EINEM ANDEREN JÜDISCHEN SELBSTVERSTÄNDNIS}

Angesichts der prominenten sowie provokativen Platzierung eines Skandals, der sich für die deutsche Erinnerungskultur um die Shoah als durchaus einschneidend erwies, wirkt diese Annahme nicht wirklich überzeugend. Auffällig ist zudem, dass Die Leinwand immerhin mehr als ein Jahrzehnt nach der Affäre um Wilkomirski und damit zu einem Zeitpunkt erschien, da hierzu eigentlich alles gesagt worden war. Es ist dies allerdings eine Phase, die in der Forschung gemeinhin als „Wendepunkt" (Assmann, Das neue Unbehagen 13) oder gar "Zäsur und Epochenschwelle“ (Frieden 28) des kollektiven Erinnerns an die Shoah gilt. Gemeint sind hiermit das vielthematisierte Sterben der letzten ZeitzeugInnen, eine damit notwendigerweise einhergehende Medialisierung der Erinnerung, gesellschaftliche Wandlungsprozesse aufgrund von Globalisierung und Migration sowie ein, aus den vorherigen Gründen resultierendes, sich veränderndes Sprechen über die Shoah innerhalb der Gesellschaft (28-35).

Die Leinwand scheint nun auf die beiden ersten Punkte, also sowohl auf das Sterben der letzten ZeugInnen als auch die Medialisierung der Erinnerung, zumindest implizit Bezug zu nehmen. Hierfür existieren im Text einige Signale: So trägt das fiktive Pendant von Wilkomirskis Bruchstücke in Steins Roman den Titel Aschentage, wodurch er - gerade im Zusammenhang mit der Frage nach der Möglichkeit authentischer Zeugenschaft - relativ offensichtlich auf Paul Celans Aschenglorie aus dessen Gedichtband Atemwende anspielt. In den letzten drei Zeilen des Gedichts findet sich die bekannte Passage: „Niemand / zeugt für den / Zeugen“ (Celan 68). In diesen Versen Paul Celans drückt sich die ganze Aporie sekundärer Zeugenschaft aus: Auf der einen Seite stellen sie einen Appell an die ZuhörerInnen dar, für das Bezeugte ein Verantwortungsgefühl zu entwickeln (Baer 7), andererseits bedeutet jenes „Zeugen für den Zeugen“ letztlich aber auch die Unterminierung der Authentizität der tatsächlichen ZeugInnen der Shoah, da „der Unterschied zwischen authentischer Erfahrung und vorgestelltem Leid, zwischen geschichtlicher Wahrheit und konstruierter Nacherzählung, zwischen Realität und Rhetorik, zwischen Fakt und Fiktion zu schwinden [droht]" (14). Der Zeuge erscheint demnach als unersetzlich. Der Intertext Aschenglorie rückt die Fiktionalisierung des Wilkomirski-Skandals in Die Leinwand somit in ein anderes Licht: Nicht die Abkehr von der Erinnerung an die Shoah ist 
das Thema, sondern ein Dilemma wird hierin angezeigt, das aus dem Sterben der jüdischen Überlebenden resultiert. Die im Roman vorgeführte Unzuverlässigkeit des individuellen Gedächtnisses erscheint solchermaßen nicht länger nur als die generelle Infragestellung der Möglichkeit von Authentizität, sondern vielmehr auch als vexierbildartiger Ausdruck einer möglichen Verunsicherung für das gegenwärtige jüdische Selbstverständnis in Hinblick auf das Erbe der Erinnerung an die Shoah. Nicht umsonst betont der Roman immer wieder die enge Verwobenheit von Gedächtnis und Identität: Welche Auswirkungen ergeben sich für die NachfahrInnen der jüdischen Überlebenden, wenn letztere die Shoah nicht mehr erinnern können? Die Figur Jan Wechslers verweist indirekt durchaus auf diese Frage: Sie steht sinnbildlich für eine unsicher gewordene Identität, nachdem die Erinnerung an das Vergangene abhandengekommen ist.

Mit Blick auf die Weitergabe der Erinnerung an die Shoah drängt sich die Frage auf, ob die Darstellung der Figur Minskys im Roman nicht auch als eine Form von Vorausdeutung auf ein sogenanntes affiliatives Postgedächtnis gelesen werden könnte. Nach Marianne Hirsch beschreibt ein solches Postgedächtnis der Shoah die Erinnerungen derjenigen, welche diese nicht selbst erlebten, sondern durch ihre Vorgängergenerationen in Erzählungen und Bildern vermittelt bekommen haben - sekundäre Erinnerungen also, in denen die Traumata, ihrem ursprünglichen Kontext entrissen und nunmehr in spezifisch geformte Repräsentationen gehüllt, überdauern. Dabei sind diese Erinnerungen keinesfalls ausschließlich familiär, sondern prinzipiell auf jede Person übertragbar, die Teil solch eines transgenerationellen Erinnerungsraums ist, dem ein kollektives Trauma zugrunde liegt (Hirsch 9-10). Die Bezugnahme auf Paul Celans Aschenglorie zeigt in diesem Zusammenhang nun allerdings die Unmöglichkeit einer so verstandenen sekundären Zeugenschaft an, da die grausame Realität der Shoah und das imaginierte Leid Minskys hier miteinander verschwimmen. Mag dessen Geschichte auch eine sozusagen therapeutische Berechtigung haben, kann sie deshalb jedoch keinesfalls die Legitimität eines Zeugnisses der Shoah für sich beanspruchen. Dergestalt könnte die provokative Infragestellung der Möglichkeit von Authentizität die spezifische Autorität der ZeugInnen paradoxerweise also noch einmal akzentuieren wollen: Gerade das komplette Aufheben der Grenzen zwischen Autobiographie und Fiktion verdeutlicht schließlich wiederum den qualitativen Unterschied zwischen einem tatsächlichen Zeugnis der Shoah und der fiktiven Erzählung Minskys. Auf diese Weise lässt sich Die Leinwand ebenso als ein nachträglicher und gewissermaßen abschließender 
Kommentar zur Ära der ZeitzeugInnen der Shoah lesen, deren Authentizität im Laufe der Jahre immer wieder zur Disposition stand - nicht zuletzt im Skandal um Wilkomirski. Durchaus unverhohlen findet sich im Roman denn auch Kritik an jenen Stimmen, die nach der Enthüllung Bruno Dössekers einen vermeintlichen „Kult des Zeugnisablegens“ (Mächler 111) beklagten: Als Jan Wechsler noch nicht ahnt, dass er selbst der Urheber des Enthüllungsbuchs Maskeraden ist, liest er verwundert vom angeblichen Geschäft „mit dem Kult ums Erinnern und um die postulierte Schuld“ (Stein W.46). Mit Blick auf den Autor fragt er ironisch:

Worauf wollte dieser Mann eigentlich hinaus? War die einzige Möglichkeit, angemessen mit dem Phänomen Auschwitz umzugehen, die, Stillschweigen zu bewahren und die Überlebenden, ohne ihnen eine Entschädigung zugebilligt zu haben, sterben zu lassen? Sollte nichts dokumentiert werden? Und wenn schon, dann ausschließlich pro bono? (W.46-47)

Die fiktionalisierte Einbettung des Wilkomirski-Falls in die besondere Form des Romans, die eine jeweils individuell gestaltbare Lektüre ermöglicht, wird indes ebenso im Lichte einer zunehmenden Medialisierung der Erinnerung an die Shoah interpretierbar - gerade auch im Kontext einer die Gesellschaft und damit auch die Erinnerungskultur immer stärker prägenden Digitalisierung. So erinnert die besondere Aufmachung von Steins Roman durchaus an einen auf Papier gedruckten Hypertext: Dessen grundsätzliches Merkmal der Non- bzw. Multilinearität, aus der die LeserInnen jeweils Kohärenz herstellen müssen, bezeichnet nämlich das Wesen der digitalisierten Textform (Simanowski par. 2). Beim Lesen eines Hypertexts werden die LeserInnen notwendigerweise dazu gezwungen, einen „Sprung“ vorzunehmen, da ihnen keine lineare, vorgegebene Grundstruktur zur Verfügung steht. Sie arbeiten somit an der Herstellung des Texts immer wieder selber mit und werden gewissermaßen zu den HerausgeberInnen ihrer eigenen Lektüreerfahrungen (Wirth 32-33). Mittels der experimentellen Anspielung auf die Form des Hypertexts fragt Die Leinwand auch danach, wie der gesellschaftliche Umgang mit sowie der Zugang zur Erinnerung sich wandeln, da diese nicht nur in ausschließlich mediatisierter Form zugänglich ist, sondern darüber hinaus im digitalen Raum auf völlig neue Weise verhandelt wird. Eben hierin macht Aleida Assmann schließlich auch eine wesentliche Ursache des von ihr diagnostizierten „Unbehagen[s] an der deutschen Erinnerungskultur“ (Assmann, Das neue Unbehagen 13) aus. Denn es stellt sich nicht zuletzt auch die Frage danach, ob die Erinnerung an die Shoah in einer Phase der 
diskursiven Fragmentierung sowie Potenzierung der Informationsflut im öffentlichen Bewusstsein an Bedeutung zu verlieren und vor allem auch im digitalen Raum zu einem „Arsenal historischer Versatzstücke“ (Wolfrum 31) zu geraten droht. Gerade solch eine versatzstückartige Montage fremder Geschichten charakterisiert ja die Figuren Jan Wechslers und Minskys im Roman. Das hervorgehobene Interesse Wechslers an Computern und den neuesten technischen Entwicklungen (Stein W.16), welches laut Paratext ebenso auf den Autor Benjamin Stein zuzutreffen scheint, ist zudem ein weiterer Hinweis dafür, dass der Roman durchaus bewusst vor dem Hintergrund medialer Wandlungsprozesse den Skandal um ein gefälschtes Zeugnis der Shoah reaktualisiert sowie reflektiert. Die wiederkehrenden Gedächtnislücken Wechslers verweisen schlussendlich auch noch einmal auf das kollektive Gedächtnis der Shoah, dessen Aufrechterhaltung angesichts der „für das WWW typischen dynamischen Beschleunigungsprozesse“ (Frieden 329) immer schwieriger werden könnte. Denn so wie ein jedes Gedächtnis auf Erinnerungen aufbaut, bedarf dieses gleichermaßen des Vergessens, um vor einer Überfülle an zu verarbeitenden Informationen bewahrt zu werden - Erinnern und Vergessen erscheinen somit als zwei unweigerlich miteinander verschränkte Vorgänge innerhalb des kollektiven Gedächtnisses (Assmann, Formen des Vergessens 11-21).

Im Zeichen dieser unsicher gewordenen Erinnerung an die Shoah ist es nun die Religiosität, die in Die Leinwand eine prägende Rolle für das jüdische Selbstverständnis einnimmt. Dabei rückt abermals die Figur Jan Wechslers in den Mittelpunkt, dessen Weg zur Observanz gegenüber dem in einem streng orthodoxen Umfeld in Israel sozialisierten Amnon Zichroni aufs engste mit dem Ablegen seiner alten Vergangenheit und dem Erschaffen einer neuen Identität verknüpft ist. Nachdem er zunehmend anerkennen muss, dass seine Erinnerungen falsch sind, berichtet Wechsler von seinem früheren Maschgiach Ariel, dem Aufseher über die jüdischen Speisegesetze: „Was ich über Torah, Talmud und die Kabbala weiß, habe ich von ihm gelernt" (Stein W.141-42). Durch Ariel begann er zunehmend damit, sein Leben immer mehr nach den religiösen Gesetzen auszurichten und Kippa, Zizit sowie später auch Tefillin zu tragen (W.145-47). Wechslers Konversion sollte schließlich durch eine Tevila, das rituelle Bad in der Mikwe, beschlossen werden, „um den Übergang in mein neues Leben auch symbolisch zu bekräftigen.“ Der Erzähler schildert in diesem Zusammenhang einen Traum, den er in der letzten Nacht seines „alten Lebens“ hatte und in dem Ariel ihn „in einem morschen Kahn über einen bleiernen Fluss stakte“ (W.147). In einer späteren 
Passage der Wechsler-Erzählung wird deutlich, dass es sich dabei um den Lethe handeln muss, der in der griechischen Mythologie den Fluss des Vergessens bezeichnet (W.188). Wechslers Konversion in der Mikwe und die Annahme seiner neuen Identität unter dem Namen Arieh Leiw geschehen hier also vor dem Hintergrund des Verlusts der alten Erinnerungen. So erklärt ihm Ariel: „Nichts würde mehr gelten von dem, was gewesen war. Aus dem Wasser steige man auf als ein neuer Mensch“ (W.148).

Doch die vergessen geglaubte Vergangenheit holt Wechsler im Laufe der Erzählung wieder ein. Dabei wird ihm ausgerechnet die Mikwe wiederum zum Verhängnis: Hier kommt es zum unaufgelösten Kampf mit Amnon Zichroni, da dieser ihm die öffentliche Verurteilung Minskys und die damit verbundene Zerstörung seiner eigenen Existenz nicht verzeihen kann (Z.193). Infolgedessen erhält Wechsler schließlich auch jenen mysteriösen Koffer, der letztlich den Bruch mit seiner Familie bewirkt und in Israel gar in seiner Festnahme mündet. Am Ende führt Wechsler die Polizei zur Mikwe bei Moza, in der Hoffnung, durch ein erneutes Eintauchen dort vielleicht wieder Einsichten in seine Vergangenheit zu erhalten - vergebens (W.203-04). Mag die Rückkehr zur Religiosität in Die Leinwand somit durchaus im Sinne des an mehreren Stellen thematisierten, kabbalistischen Tikkun olam verhandelt werden, ,einer langsamen, mühsamen, kollektiven Verantwortung für die Vervollkommnung der Welt" (Kleine 187), so funktioniert dies jedoch keinesfalls durch die komplette Abwendung von der Erinnerung - dies stellt der scheiternde Jan Wechsler unter Beweis. Stattdessen spielt das Überqueren des Lethe hier vielmehr auf jene „Epochenschwelle" an, an der sich die Erinnerung an die Shoah befindet. Hieraus ergeben sich zum einen die Frage nach einer verantwortungsvollen Weitergabe des Erinnerten, andererseits möglicherweise aber auch nach der Suche eines alternativen jüdischen Selbstverständnisses. „Ein befreundeter Autor hat mir Jahre später anvertraut, dass seine jüdische Identität auch in Westdeutschland nur zwei Anker hatte: Israel und Auschwitz" (Stein W.130), heißt es in der Wechsler-Erzählung. In Die Leinwand stellen die Hinwendung zu einem orthodoxen Lebensstil und der Kabbala ein mögliches Identitätsangebot abseits dessen dar. 


\section{ÄSTHETISCHE REFLEXION \\ EINES THEORETISCHEN METADISKURSES}

Der Versuch einer Einordnung von Benjamin Steins Die Leinwand in Hinblick auf die Rolle der Erinnerung an die Shoah im Roman kann allerdings nicht umhinkommen, abschließend wenigstens kurz auf dessen komplexe literarische Reflexion postmoderner Theoriebildungen hinzuweisen. Vor dem Hintergrund der Veränderungen des gegenwärtigen kollektiven Gedächtnisses nimmt der Text anhand der Problematisierung des Verhältnisses von Erinnerung und Authentizität sowie von Fiktion und Autobiographie hier tatsächlich nicht zuletzt auch eine ästhetische Evaluierung eines - in sich gewiss heterogenen - Theoriediskurses vor, der infolge des linguistic turn nicht nur die Geisteswissenschaften und ihre einzelnen Disziplinen fundamental und dauerhaft verändern sollte - durch die Erkenntnis nämlich, dass, zugespitzt formuliert, Wirklichkeit außerhalb von Sprache nicht vorhanden sein kann. So lassen sich die Frage nach der Möglichkeit zuverlässigen Erinnerns sowie die provokative Infragestellung jeglichen autobiographischen Anspruchs durchaus auf das poststrukturalistische Kernpostulat zurückführen, wonach ein den Signifikanten vorausgehendes Signifikat nicht existent oder vorstellbar ist: „Das Substitut ersetzt nichts, das ihm irgendwie präexistiert hätte“ (Derrida 117). Demgemäß ließe sich Wirklichkeit also nicht durch einen nachträglichen Akt der Versprachlichung abbilden, sondern jene konstituiert sich überhaupt erst in diesem. Gerade im Zusammenhang mit der Fiktionalisierung des Skandals um ein gefälschtes Zeugnis der Shoah verhandelt Die Leinwand somit zumindest implizit das Dilemma, wonach die Lossagung des Zeichens und seiner Gültigkeit von jeglicher außersprachlichen Referenz in letzter Konsequenz auch die Gefahr eines beliebigen Überschreibens erfahrener unmenschlicher Realität in den Konzentrations- und Vernichtungslagern nach sich zieht. So sieht auch Alessandro Costazza Steins Roman in der Tradition des linguistic turn verortet und hinterfragt kritisch dessen vermeintliche Gleichsetzung von Fiktion und Autobiographie (Costazza 330-31). Allerdings geht Costazza dabei von einer eindeutigen Parteinahme des Buchs für Wilkomirski aus (328), anstatt von einer Inszenierung postmoderner Aporien, aus der letztlich auch ein Moment der Kritik erwachsen kann. Es ergeben sich aus der Lektüre von Steins Roman jedenfalls fraglos Anknüpfungspunkte an eine Debatte, die bereits vor einigen Jahrzehnten die Geschichtswissenschaft mit Blick auf die historische Aufarbeitung der Shoah heimsuchte und von Saul Friedlander wie folgt zu- 
sammengefasst wurde: "[P] ostmodern thought's rejection on the possibility of identifying some stable reality or truth beyond the constant polysemy and self-referentiality of linguistic construct challenges the need to establish the realities and the truths of the holocaust $[\ldots]$ " (Friedlander 4-5).

So geriet bezeichnenderweise gerade der Skandal um Binjamin Wilkomirski zum Exempel dafür, welche problematischen Konsequenzen sich aus der „Löschung des Unterschieds von ,Leben“ und ,geschriebenem Leben“" (Janser und Kilchmann 113) ergeben: Schließlich war es nicht nur das Feuilleton, das den fiktiven Charakter von Bruchstücke nicht bemerkte, sondern vor allem auch ein Großteil desjenigen wissenschaftlichen Diskurses, der sich mit den Zeugnissen Überlebender der Shoah auseinandersetzte. (Oels 381) Zum Ausdruck kam hier eine wohl keinesfalls nur zufällige Ähnlichkeit zwischen der „postmodernen“ Relativierung außersprachlicher Wirklichkeit auf der einen und einer auffälligen Selbstreferenzialität in der medialen Debatte um Wilkomirski auf der anderen Seite, wie sie häufig im Laufe von Diskussionen um die Erinnerungskultur der Shoah zutage tritt (Zuckermann 99). Während es vor allem darum zu gehen schien, „das wahre Gedenken, und das ist freilich immer das selbst praktizierte, gegen das falsche von den anderen geübte, auszuspielen“ (Oels 379), trat die Befassung mit dem eigentlich zu Erinnernden hingegen in den Hintergrund.

Benjamin Steins Die Leinwand scheint also nicht nur den Wandel des kollektiven Gedächtnisses und dessen Auswirkungen auf das jüdische Selbstverständnis in Deutschland zu reflektieren, sondern ließe sich zugleich auch als ein gewissermaßen ästhetischer Kommentar zur Tradition postmoderner Theoriebildungen verstehen. Mehr noch: Hieraus entwickelt der Roman überhaupt erst sein kritisches Potential mit Blick auf den Erinnerungsdiskurs um die Shoah. Dabei knüpft der Text mittels seiner besonderen Form durchaus an die Tendenz eines ästhetischen Avantgardismus innerhalb der jüngeren deutsch-jüdischen Literatur an. Mag Die Leinwand anfänglich als literarische Provokation erscheinen, für die Auschwitz keine wichtige Rolle mehr einnimmt, so erweist sich diese Geste im weiteren Verlauf der Lektüre demgegenüber vielmehr als implizite Aufforderung zu einer verantwortungsvollen Auseinandersetzung mit dem Erbe der Erinnerung, während nicht zuletzt auch der Aspekt des Religiösen wieder Einzug in die deutschsprachigjüdische Literatur erhält. 


\section{ZITIERTE WERKE}

PRIMÄRTEXTE

Celan, Paul. Atemwende. 1967. Suhrkamp Taschenbuch, 1982.

Stein, Benjamin. Die Leinwand. 2010. dtv, 2015.

Wilkomirski, Binjamin. Bruchstücke. Aus einer Kindheit 1939-1948. Jüdischer Verlag, 1995.

\section{FORSCHUNGSLITERATUR}

Assmann, Aleida. Das neue Unbehagen an der Erinnerungskultur. Eine Intervention. C.H. Beck, 2013.

Assmann, Aleida. Formen des Vergessens. Wallstein Verlag, 2016.

Baer, Ulrich. Einleitung. "Niemand zeugt für den Zeugen". Erinnerungskultur und historische Verantwortung nach der Shoah, edited by Ulrich Baer, Suhrkamp, 2000, pp. 7-31.

Basseler, Michael, and Dorothee Birke. Mimesis des Erinnerns. Gedächtniskonzepte der Literaturwissenschaft. Theoretische Grundlegung und Anwendungsperspektiven, edited by Astrid Erll and Ansgar Nünning, de Gruyter, 2005, pp. 123-47.

Costazza, Alessandro. "Benjamin Steins Die Leinwand oder über die (Un-)Möglichkeit (auto)biographischen Schreibens." Logik der Prosa: Zur Poetizität ungebundener Rede, edited by Astrid Arndt, Christoph Deupmann and Lars Körten, V \& R unipress, 2012, pp. 301-33.

De Man, Paul. “Autobiographie als Maskenspiel.” 1979. Die Ideologie des Ästhetischen, edited by Christoph Menke, Suhrkamp, 1993, pp. 131-46.

Derrida, Jacques. "Die Struktur, das Zeichen und das Spiel im Diskurs der Wissenschaften vom Menschen.” 1967. Postmoderne und Dekonstruktion. Texte französischer Philosophen der Gegenwart, edited by Peter Engelmann, Reclam, 1990, pp. 114-39.

Diner, Dan. "Negative Symbiose. Deutsche und Juden nach Auschwitz." 1986. Ist der Nationalsozialismus Geschichte? Zu Historisierung und Historikerstreit, edited by Dan Diner, Fischer, 1993, pp. 185-97.

Düwell, Susanne. "Fiktion aus dem Wirklichen." Strategien autobiographischen Erzählens im Kontext der Shoah. Aisthesis Verlag, 2004.

Finck, Almut. Autobiographisches Schreiben nach dem Ende der Shoah. Erich Schmidt Verlag, 1999. Geschlechterdifferenz \& Literatur 9.

Frieden, Kirstin. Neuverhandlungen des Holocaust. Mediale Transformationen des Gedächtnisparadigmas. transcript Verlag, 2014. Erinnerungskulturen / Memory Cultures 3.

Friedlander, Saul. "Introduction." Probing the Limits of Representation. Nazism and the "Final Solution," edited by Saul Friedlander, Harvard UP, 1992, pp. 1-21.

Garloff, Katja. "The Power of Paratext: Jewish Authorship and Testimonial Authority in Benjamin Stein's Die Leinwand." Persistence Legacy. The Holocaust and German Studies, edited by Erin McGlothlin and Jennifer M. Kapczynski, Camden House, 2016, pp.141-55.

Garloff, Katja, and Agnes Mueller. "Introduction." German Jewish Literature after 1990, edited by Katja Garloff and Agnes Mueller, Camden House, 2018, pp. 1-16.

Günter, Manuela. Überleben schreiben. Zur Autobiographik der Shoah. Königshausen \& Neumann, 2002. 
Hessing, Jakob. “Aufbrüche. Zur deutsch-jüdischen Literatur seit 1989.” Handbuch der deutschjüdischen Literatur, edited by Hans Otto Horch, de Gruyter, 2015, pp. 244-69.

Hirsch, Marianne. "Surviving Images. Holocaust Photographs and the Work of Postmemory." The Yale Journal of Criticism, vol. 14 no. 1, 2001, pp. 5-27.

Hoffmann, Daniel. "Deutsch-jüdische religiöse Literatur in der Moderne." Handbuch der deutschjüdischen Literatur, edited by Hans Otto Horch, de Gruyter, 2015, pp. 435-47.

Horstkotte, Silke. „Ich bin, woran ich mich erinnere.“ Benjamin Steins Die Leinwand und der Fall Wilkomirski. Der Nationalsozialismus und die Shoah in der deutschsprachigen Gegenwartsliteratur, edited by Torben Fischer, Philipp Hammermeister and Sven Kramer, Rodopi, 2014, pp. 115-32. Amsterdamer Beiträge zur neueren Germanistik 84.

Janser, Daniela, and Esther Kilchmann. "Der Fall Wilkomirski und die ,condition postmoderne'." Traverse: Zeitschrift für Geschichte = Revue d'histoire, vol. 7, no. 3, 2000, pp. 108-21.

Kilcher, Andreas B. "Exterritorialitäten. Zur kulturellen Selbstreflexion der aktuellen deutschjüdischen Literatur." Deutsch-jüdische Literatur der neunziger Jahre. Die Generation nach der Shoah, edited by Sander L. Gilman and Hartmut Steinecke, Erich Schmidt Verlag, 2002, pp. 131-46. Beihefte zur Zeitschrift für deutsche Philologie 11.

Kilian, Arne. "Wilkomirski-Affäre.” Lexikon der ,,Vergangenheitsbewältigung “ in Deutschland. Debatten- und Diskursgeschichte des Nationalsozialismus nach 1945, edited by Torben Fischer and Matthias N. Lorenz, transcript, 2015.

Kleine, Johannes. Islam und Judentum in der deutschsprachigen Gegenwartsliteratur. Zur Bedeutung religiöser Elemente in ausgewählten Werken von Benjamin Stein und Navid Kermani. n.p., 2017.

Koelle, Lydia. "Identität und Konvektion - Erbe oder Eskapismus? Die Nachkommen der Überlebenden und der Kriegskinder erschreiben sich ihren Ort und ihre Zeit." Deutschjüdische Identität. Mythos und Wirklichkeit. Ein neuer Diskurs?, edited by Norbert Honsza and Przemyslaw Sznurkowski, Peter Lang, 2013, pp. 209-31. Polnische Studien zur Germanistik, Kulturwissenschaft und Linguistik 5.

Lejeune, Philippe. Der autobiographische Pakt. 1975. Suhrkamp, 1994.

Mächler, Stefan. “Aufregung um Wilkomirski. Genese eines Skandals und seine Bedeutung.” Das Wilkomirski-Syndrom. Eingebildete Erinnerungen oder Von der Sehnsucht, Opfer zu sein, edited by Irene Diekmann and Julius H. Schoeps, Pendo, 2002, pp. 86-131.

Michaelis, Andree. Erzählräume nach Auschwitz. Literarische und videographierte Zeugnisse von Überlebenden der Shoah. Akademie Verlag, 2013. WeltLiteraturen / World Literatures 2.

Michaelis-König, Andree. "Multilingualism and Jewishness in Katja Petrowskaja's Vielleicht Esther." German Jewish Literature after 1990, edited by Katja Garloff and Agnes Mueller, Camden House, 2018. 146-66.

Neumann, Birgit. "Literatur, Erinnerung, Identität." Gedächtniskonzepte der Literaturwissenschaft. Theoretische Grundlegung und Anwendungsperspektiven, edited by Astrid Erll and Ansgar Nünning, de Gruyter, 2005, pp. 149-78.

Oels, David. “,A real-life Grimm’s fairy tale. Korrekturen, Nachträge, Ergänzungen zum Fall Wilkomirski.” Zeitschrift für Germanistik, Neue Folge, vol. 14, no. 2, 2004, pp. 373-90.

Simanowski, Roberto. "Hypertext: Merkmale, Forschung, Poetik." dichtung-digital, vol. 24, 2002): n. pag. www.dichtung-digital.de/2002/07-31-Simanowski.htm. Accssed 28 June 2019. 
Schmidt, Sibylle, and Ramon Voges. "Einleitung." Politik der Zeugenschaft. Zur Kritik einer Wissenspraxis, edited by Sybille Krämer, Sibylle Schmidt, and Ramon Voges, transcript, 2011, pp. 7-20.

Schuchmann, Kathrin. “,Unser Gedächtnis ist der wahre Sitz unseres Ich. ' Erinnerung und Geschichte(n) in Benjamin Steins Die Leinwand.” Zagreber Germanistische Beiträge 21 (2013): 201-20.

Wirth, Uwe. "Wen kümmert's, wer spinnt?" Hyperfiction. Hyperliterarisches Lesebuch: Internet und Literatur, edited by Beat Suter and Michael Böhler, Stroemfeld/Nexus, 1999, pp. 29-42.

Wolfrum, Edgar. "Erinnerungskultur und Geschichtspolitik als Forschungsfelder." Reformation und Bauernkrieg. Erinnerungskultur und Geschichtspolitik im geteilten Deutschland, edited by Jan Scheunemann, Evangelische Verlagsanstalt, 2010, pp. 13-47.

Zuckermann, Moshe. Gedenken und Kulturindustrie. Ein Essay zur neuen deutschen Normalität. Philo, 1999.

\section{POWIEŚĆ DIE LEINWAND BENJAMINA STEINA: \\ POWRÓT RELIGIJNOŚCI ŻYDOWSKIEJ POD ZNAKIEM NIEPEWNEJ PAMIĘCI O ZAGŁADZIE}

\section{Streszczenie}

Powieść Die Leinwand Benjamina Steina fikcjonalizuje skandal wokół literackiego fałszerstwa autorstwa Binjamina Wilkomirskiego, którego tekst, ogłoszony jako świadectwo Szoah, okazał się być fikcyjną opowieścią. Die Leinwand w prowokacyjny sposób problematyzuje wiarygodność wspomnień i pyta o granice między autobiografią a fikcją. W artykule podkreślono, że te strategie estetyczne powinny być interpretowane w kontekście odchodzenia ostatnich świadków i wynikającej z tego faktu konieczności mediatyzacji pamięci o Zagładzie. W świetle tych zmian w powieści następuje powrót do religijnego aspektu tożsamości żydowskiej. Krytyczny potencjał powieści, ze zwrotem ku dyskursowi pamięci, stanowi przede wszystkim refleksja literacka dotycząca teorii postmodernistycznych.

Słowa kluczowe: Benjamin Stein; pamięć zbiorowa o Szoah; religijność żydowska; postmodernizm.

\section{BENJAMIN STEIN'S NOVEL THE CANVAS: \\ THE RETURN OF JEWISH RELIGIOSITY UNDER THE SHADOW OF THE UNCERTAIN MEMORY OF THE SHOAH}

\section{S u m m a ry}

Benjamin Stein's novel The Canvas fictionalises the literary scandal around Binjamin Wilkomirski, whose supposed testimony of the Shoah proved to be fiction. The Canvas thereby provocatively questions the reliability of memories, as well as the differences between autobiographies and fiction in general. This paper argues that those aesthetic strategies have to be interpreted in the context of the passing of the last witnesses and thus of the necessary medialisation of the memory of the Shoah. In the light of those transformations, it is the religious aspect of Jewish identity which is foregrounded in the novel. The Canvas's critical view of the cultural memory discourse is based in particular on a literary reflection of postmodern theorising.

Key words: Benjamin Stein; culture of memory; collective memory of the Shoah; Jewish religiousness; postmodernism. 